\title{
TP53 status in Mantle Cell Lymphoma (MCL) - A 10- year single center experience
}

\author{
Elhassadi $E^{*}$, Hennessy $B^{1}$, Kumar $S^{1}$, Swan $D^{1}$, Lee $E^{2}$, Barrett ${ }^{1}$, Doyle $M^{2}$, Shilling $C^{2}$ and Catherwood $M^{3}$ \\ ${ }^{1}$ Haematology Department, University Hospital Waterford, Ireland \\ ${ }^{2}$ Pathology Department, University Hospital Waterford, Ireland \\ ${ }^{3}$ Molecular Department, Belfast University Hospital, Ireland
}

Mantle Cell Lymphoma (MCL) is a rare sub-type of B-cell nonHodgkin lymphoma (NHL), with variable course. Most of patients with this disease have an aggressive clinical course and poor prognosis. However, a minority of patient may survive untreated for many years. MCL is responsive to a variety of initial therapies, but relatively short-term remissions are achieved with conventional chemotherapy regimens. The median duration of remission in most trials using standard chemo-immunotherapy is $1.5-3$ years and the median overall survival (OS) is 3-6 years. TP53 mutation confers a dismal prognosis in MCL with a median survival of 1.3 years $[1,2]$.

We reporting our single centre retrospective study on MCL patients, treated over 10 years (2006-2016), reflects a real-life practice outside clinical trial context. The study was carried out to examine the impact of TP53 mutational status in our cohort treatment out-come. Also, to correlate the $\mathrm{p} 53$ expression using immuno-histochemistry (IHC) with mutational status. The median age was 65 years with male predominance (72\%), and the majority of patients had advanced disease (90\%) with a high MIPI score in $60 \%$ of the cases, $9 \%$ had a prior history of an indolent leukemic sub-type which evolved subsequently to aggressive disease requiring intervention. 13 (59\%) patients had mutated $I G H V$, $9(41 \%)$ with unmutated genes, no residual tissue in the reminder. p53 over-expression $(>30 \%)$ seen in $6(21 \%)$ samples, Sanger sequencing (SS) detected TP53 mutations only in $3(10 \%)$ samples. The remaining samples were subjected to next generation sequencing (NGS) to overcome SS sensitivity limitation. Interestingly, NGS analysis revealed presence of mutations in all 6 samples (100\%), confirming existing of sub-clones beyond of SS detection sensitivity. In contrast, all samples with low p53 expression, sequencing confirmed wild-type TP53 status suggestive of high negative predictive value and higher sensitivity of IHC. These finding reflective of a strong correlation between p53 expression by IHC and mutation status. Sequencing analysis for all samples $(\mathrm{N}=32)$ confirmed the presence of a deleterious mutation in $6(21 \%)$ samples which is comparable with reported literature $[1,3]$.

Chemo-immunotherapy remains the main treatment modality in our cohort, resulted in an ORR of $79 \%$, CR $55 \%$ and PR $24 \%$, which compares well with the reported literature [4]. $6(21 \%)$ patients were eligible for ASCT, only 2 (7\%) patient had received ASCT consolidation resulted in CR, while the remainder (4 patients $=14 \%$ ) had failed stem cell mobilization and maintained their initial treatment response. Although, HCVAD treated patients (2 patients, $7 \%$ ), with mutated TP53, retained durable CR, the number of cases is small to extrapolate this approach could overcome the impact of this genetic aberrations in MCL. 15 (52\%) patients received Rituximab maintenance (RM), including 2 (7\%) patients in which it was used post-ASCT. The majority
(80\%) of RM patients have maintained their treatment response. Thirteen $(45 \%)$ of our patients had relapsed after initial treatment including $1(3 \%)$ patient with HL relapse. The median duration of response prior to relapse was 19 months (range 9 - 36 months).

At relapse (13 patients), chemo-immunotherapy used as second line therapy in $7(24 \%)$ patients resulted in CR in 5 (71\%) patients and $2(29 \%)$ patients with progressive disease subsequently treated with Ibrutinib as third line therapy. Five (17\%) relapse patients treated with Ibrutinib as second line therapy, as third line therapy in 2 (7\%) patients and $1(3 \%)$ patient was unfit for any further therapy. Most patients on Ibrutinib therapy obtained a durable response, with 4 (14\%) patients maintained their response to the study date while the reminder $(3$ patients $=10 \%)$ relapsed with progressive disease. $1(3 \%)$ patients received allo-SCT consolidation for relapsed disease resulted in transplant related mortality (TRM) due to severe Graft versus host disease (GVHD). The total number of deaths over the study period were $11(38 \%)$ patients, lymphoma related death were accounted in 5 (45\%) patients. Of these, $4(80 \%)$ patients were harbouring deleterious TP53 mutations and the remaining patient (20\%) with multiple relapse disease, his relapsed biopsy showed high expression p53 expression but no residual tissue available to carry out TP53 sequencing (Table 1). These findings confirm the dismal outcome of MCL patients harbouring TP53 mutations as reported in literature. In contrast, the remaining (6=patients / 55\%) were non-lymphoma related death including 5 (17\%) patients due to extreme age and co-morbidities including second malignancy, and $1(3 \%)$ patient TRM due to severe GVHD

The median overall survival (OS) and progression free survival (PFS) for the entire cohort were 6.5 and 3 years respectively (Figure 1). Most of MCL cases harbouring TP53 mutations have an aggressive disease course with multiple disease relapses and lymphoma related deaths (80\%). In sub-set analyses, the OS was only 4 years in TP53 mutated disease and was not reached in wild-type TP53 cohort ( $P$ value $=0.007, \log$ rank test) which is comparable to the reported data (Figure 2).

Optimal management of MCL patient's harbouring TP53 mutation is un-met clinical need, and alternative therapeutic approaches independent of the TP53 pathway should be considered. Despite a significant improvement in disease outcomes achieved by the addition of Rituximab and high-dose Cytarabine to chemotherapy regimes,

${ }^{\star}$ Correspondence to: Elhassadi E, Haematology Department, Department, University Hospital Waterford, Ireland, E-mail: ezzat.elhassadi@hse.ie

Received: June 21, 2019; Accepted: July 16, 2019; Published: July 19, 2019 
Table 1. Study Cohort p53 IHC \& mutation sequencing results

\begin{tabular}{|l|c|c|}
\hline \multicolumn{1}{|c|}{ Value } & $\%$ \\
\hline P53\% by IHC (N=29) Pos vs Neg & 6 vs 23 & 21 vs $79 \%$ \\
\hline TP53 screening by Sanger Seq (ss) (N=29)* Pos vs Neg & 3 vs 26 & 10 vs $90 \%$ \\
\hline TP53 screening by NGS (N=3) p53 pos and SS neg samples & All positive & $100 \%$ \\
\hline Entire cohort mutational screening (N=29)* Pos vs Neg & 6 vs 23 & 21 vs $79 \%$ \\
\hline
\end{tabular}

OS and PFS

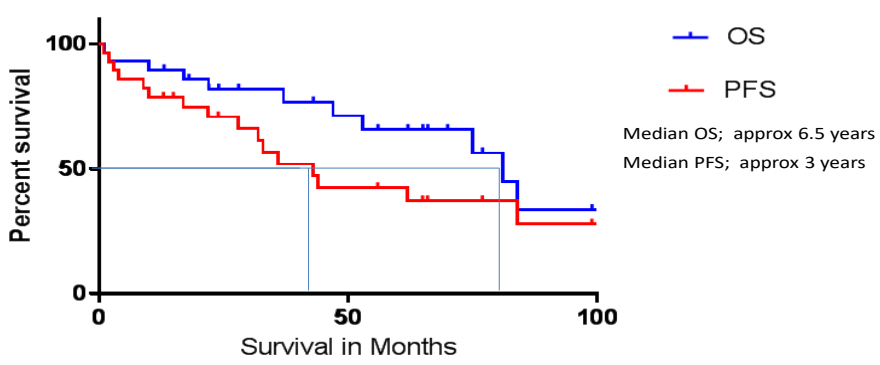

Figure 1. Study Cohort PFS \& OS KM curves

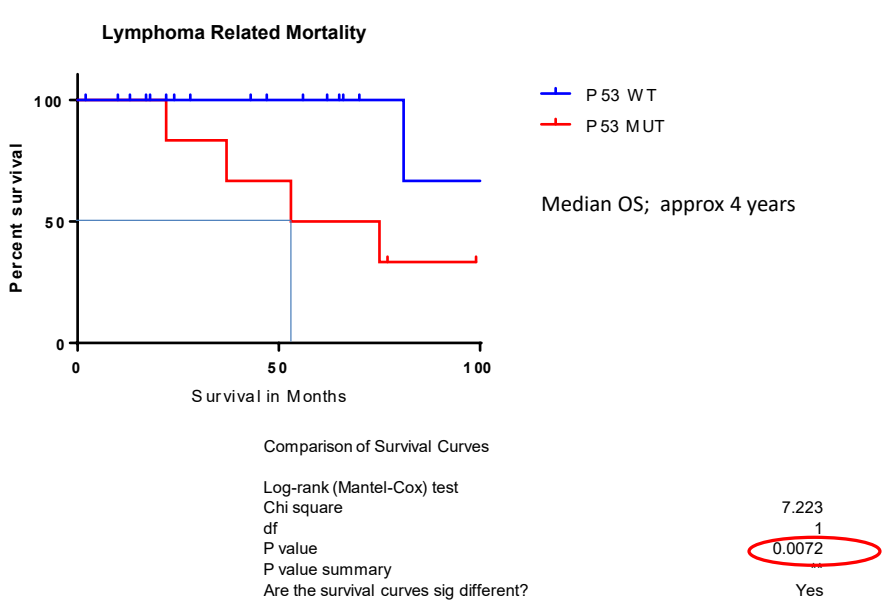

Figure 2. TP53 mutation impact KM curves and consolidation with ASCT, which is considered to be the current standard of care for younger patients, this approach dose not solves the problem of TP53 disruption for MCL patients [5,6].

The increasing number of therapeutic options is opening new perspectives (BCR inhibitors [7]. BCL2 Inhibitors [8], immunotherapy [9] for MCL patients but the evaluation of these approaches will require a correct stratification of the patients according to the specific biological risk of their disease. Screening for TP53 disruption is now included in chronic lymphocytic leukaemia (CLL) prior treatment workup for prognostic and therapeutic decision [10]. This is not altogether surprising, even in MCL where the risk of genomic instability is greater, as conventional chemo-immunotherapy relies on an intact TP53 pathway. It seems logical that the management of MCL patients might benefit from a similar approach.

\section{References}

1. Greiner TC, Moynihan MJ, Chan WC, Lytle DM, Pedersen a, et al. (1996) P53 Mutations in Mantle Cell Lymphoma Are Associated With Variant Cytology and Predict a Poor Prognosis. Blood 87: 4302-4310.

2. Dreyling MH, Bullinger L, Ott G, Stilgenbauer S, Müller-Hermelink HK, et al. (1997) Alterations of the cyclin D1/p16-pRB pathway in mantle cell lymphoma. Cancer Research 57: 4608-4614.

3. Halldórsdóttir AM, Lundin A, Murray F, Mansouri L, Knuutila S, et al. (2011) Impact of TP53 mutation and 17p deletion in mantle cell lymphoma. Leukemia 25: 1904-1908. [Crossref]

4. Dreyling M, Geisler C, Hermine O, Kluin-Nelemans HC, Le Gouill S, et al. (2014) Newly diagnosed and relapsed mantle cell lymphoma: ESMO clinical practice guidelines for diagnosis, treatment and follow-up. Annals of Oncology 25: iii83-iii92.

5. Delfau-Larue MH, Klapper W, Berger F, Jardin F, Briere J, et al. (2015) High-dose cytarabine does not overcome the adverse prognostic value of CDKN2A and TP53 deletions in mantle cell lymphoma. Blood 126: 604-611.

6. Eskelund CW, Kolstad A, Jerkeman M, Raty R, Laurell A, et al. (2016) 15-year followup of the Second Nordic Mantle Cell Lymphoma trial (MCL2): prolonged remissions without survival plateau. Br J Haematol 175: 410-418.

7. Wang ML, Rule S, Martin P, Goy A, Auer R, et al. (2013) Targeting BTK with ibrutinib in relapsed or refractory mantle-cell lymphoma. $N$ Engl J Med 369: 507-516. [Crossref]

8. Tam CS, Anderson MA, Pott C, Agarwal R, Handunnetti S, et al. (2018) Ibrutinib plus Venetoclax for the Treatment of Mantle-Cell Lymphoma. N Engl J Med 378: 12111223. [Crossref]

9. Curran KJ, Pegram HJ, Brentjens RJ (2012) Chimeric antigen receptors for T cel immunotherapy: current understanding and future direction. J Gene Med 14: 405-415.

10. Zenz T, Vollmer D, Trbusek M, Smardova J, Benner A, et al. (2010) TP53 mutation profile in chronic lymphocytic leukemia: Evidence for a disease specific profile from a comprehensive analysis of 268 mutations. Leukemia 24: 2072-2079.

Copyright: (C2019 Elhassadi E. This is an open-access article distributed under the terms of the Creative Commons Attribution License, which permits unrestricted use, distribution, and reproduction in any medium, provided the original author and source are credited. 